\title{
Gap between clinical and basic researches in multifocal synchronous lung adenocarcinoma studies
}

\author{
Yoshihisa Shimada, Norihiko Ikeda \\ Department of Thoracic Surgery, Tokyo Medical University, Tokyo, Japan \\ Correspondence to: Yoshihisa Shimada, MD, PhD. Department of Thoracic Surgery, Tokyo Medical University, 6-7-1, Nishi Shinjuku, Shinjuku-ku, \\ Tokyo 160-0023, Japan. Email: zenkyu@za3.so-net.ne.jp. \\ Provenance: This is an invited Editorial commissioned by the Section Editor Min Zhang (Department of Thoracic Oncology, The First Affiliated \\ Hospital of Chongqing Medical University, Chongqing, China). \\ Comment on: Gao RW, Berry MF, Kunder CA, et al. Survival and risk factors for progression after resection of the dominant tumor in multifocal, \\ lepidic-type pulmonary adenocarcinoma. J Thorac Cardiovasc Surg 2017;154:2092-9.e2.
}

Submitted Sep 04, 2018. Accepted for publication Sep 14, 2018.

doi: $10.21037 /$ jtd.2018.09.89

View this article at: http://dx.doi.org/10.21037/jtd.2018.09.89

At present, lepidic-type adenocarcinoma of the lung has been frequently encountered in daily clinical practice. These tumors manifest persistent pure or partial ground glass opacity (GGO) on high resolution computed tomography (HRCT) and have less recurrence if complete resection is achieved. Extensive research has investigated molecular and clinical characterization of lung adenocarcinoma, with marked success in identifying distinctive clinical features and specific molecular cohorts of patients. One of these marked clinical features is that lepidic-type adenocarcinomas are sometimes detected as multiple lesions. Two major clinical questions for patients in whom multifocal nodules present are: (I) whether synchronous multifocal lung cancers represent distinct primary tumors or intrapulmonary metastasis; and (II) how multifocal GGO should be managed when physicians consider them to be different multiple lung cancers. Gao et al. in this issue of The Fournal of Thoracic and Cardiovascular Surgery cast new light on these topics, especially the latter, and the results of the study raise both important biological and clinical management questions (1).

As the basis for their study, Gao et al. examined 70 patients who underwent resection of a $\mathrm{pN} 0$, lepidiccontaining adenocarcinoma as the resected dominant tumor (DT), who harbored at least 1 additional GGO, identified factors associated with progression of unresected GGOs, and investigated prognostic impact of growth of satellite lesions and intervention for a progressing GGO (1). It turned out that greater DT size and an initial GGO $>1 \mathrm{~cm}$ was the only factors independently associated with GGO progression while neither the progression of GGOs nor progression-prompting intervention influenced survival (1). Recent several reports that addressed prognostic determinants in multifocal GGOs revealed that they did not behave T4 or M1a advance diseases and survival of patients with multifocal GGOs was strongly affected by radiological findings of a DT $(2,3)$. These data suggest that a DT dominates prognosis as well as the progression of satellite GGOs, and strict surgical control for a DT should be most crucial and reasonable. We also encountered multifocal pure GGO cases which can not be separated into a DT and others in daily practice. On these cases, Kim et al. suggest that close follow-up without any interventions would be acceptable since the size of most focal pure GGOs associated with lung cancer does not change during long term follow-up period (4). In any case, a surgical strategy for multifocal GGOs that we have learned from Gao and colleague's report is that surgeons should primarily focus on a DT and can allow GGOs to remain without any interventions when they can be separated into a DT and other satellite nodules by tumor size or distinctive radiographic features such as bearing solid areas.

However, if we try to assemble the results and evidence from the aforementioned clinical studies as to multifocal GGOs, the appropriate timing to provide some interventions and surveillance methods for satellite 
lesions still remain to be clarified. Moreover, we do not really know multifocal pure GGO lesions and multiple GGOs with a DT should be distinguished in implications in disease pathogenesis, and whether the concept of a field cancerization phenomenon in which carcinogenic exposure or genetic factors affect tissues and organs can be applied to the key molecular mechanism by which multifocal GGO lesions arise $(5,6)$. Do the findings shown in the manuscript by Gao et al. indicate that a DT would represent an inducer of multistep tumorigenesis, in which tumors evolve through sequential progressive pathologic changes with corresponding genetic and epigenetic aberrations? The more we accumulate the relevant clinical data, the wider range of questions relating multiple synchronous lung cancer's pathophysiology arises. In other words, speculation and knowledge for biological process and molecular mechanism of multifocal lung cancer is warranted to bridge the clinical-basic research gap. Recent genomic analyses addressed the issue of genomic heterogeneity in multifocal lung cancer and revealed independent clonality of synchronous lesions in each individual and profound genomic heterogeneity at both interfocal and intrafocal levels $(7,8)$, and multifocal GGO lesions may represent the simultaneous occurrence of multistep tumorigenesis in the same lobe or bilateral lungs. Advances in understanding pathogenesis of multifocal GGOs from both biological and clinical points of view may enable us to gain important and novel insights into the molecular mechanism of the origin of multifocal lung cancers, and subsequent development of unique biomarkers for early detection of a certain cohort of GGOs that needs to be treated promptly and possibly personalized prevention tactics.

\section{Acknowledgements}

None.

Cite this article as: Shimada Y, Ikeda N. Gap between clinical and basic researches in multifocal synchronous lung adenocarcinoma studies. J Thorac Dis 2018;10(Suppl 33):S4021-S4022. doi: 10.21037/jtd.2018.09.89

\section{Footnote}

Conflicts of Interest: The authors have no conflicts of interest to declare.

\section{References}

1. Gao RW, Berry MF, Kunder CA, et al. Survival and risk factors for progression after resection of the dominant tumor in multifocal, lepidic-type pulmonary adenocarcinoma. J Thorac Cardiovasc Surg 2017;154:2092-9.e2092.

2. Shimada Y, Saji H, Otani K, et al. Survival of a surgical series of lung cancer patients with synchronous multiple ground-glass opacities, and the management of their residual lesions. Lung Cancer 2015;88:174-80.

3. Gu B, Burt BM, Merritt RE, et al. A dominant adenocarcinoma with multifocal ground glass lesions does not behave as advanced disease. Ann Thorac Surg 2013;96:411-8.

4. Kim HK, Choi YS, Kim J, et al. Management of multiple pure ground-glass opacity lesions in patients with bronchioloalveolar carcinoma. J Thorac Oncol 2010;5:206-10.

5. Kadara H, Wistuba II. Field cancerization in non-small cell lung cancer: Implications in disease pathogenesis. Proc Am Thorac Soc 2012;9:38-42.

6. Gazdar AF, Minna JD. Multifocal lung cancers--clonality vs field cancerization and does it matter? J Natl Cancer Inst 2009;101:541-3.

7. Liu Y, Zhang J, Li L, et al. Genomic heterogeneity of multiple synchronous lung cancer. Nat Commun 2016;7:13200.

8. Ma P, Fu Y, Cai MC, et al. Simultaneous evolutionary expansion and constraint of genomic heterogeneity in multifocal lung cancer. Nat Commun 2017;8:823. 